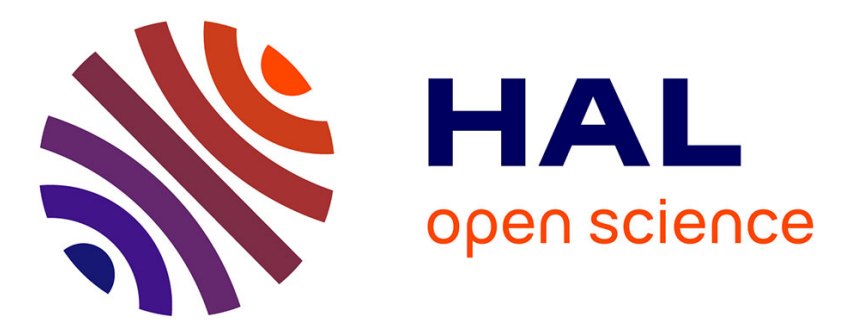

\title{
Le gel des avoirs d'une banque centrale étrangère comme réaction décentralisée à un fait internationalement illicite : rétorsion ou contremesure? \\ Emanuel Castellarin
}

\section{- To cite this version:}

Emanuel Castellarin. Le gel des avoirs d'une banque centrale étrangère comme réaction décentralisée à un fait internationalement illicite: rétorsion ou contremesure?. Hague Yearbook of International Law / Annuaire de La Haye de Droit International, Vol. 25 (2012), 25, 2012, 978-90-04-25880-8. hal-03199767

\section{HAL Id: hal-03199767 \\ https://hal.science/hal-03199767}

Submitted on 26 Apr 2021

HAL is a multi-disciplinary open access archive for the deposit and dissemination of scientific research documents, whether they are published or not. The documents may come from teaching and research institutions in France or abroad, or from public or private research centers.
L'archive ouverte pluridisciplinaire HAL, est destinée au dépôt et à la diffusion de documents scientifiques de niveau recherche, publiés ou non, émanant des établissements d'enseignement et de recherche français ou étrangers, des laboratoires publics ou privés. 


\title{
Le gel des avoirs d'une banque centrale étrangère comme réaction décentralisée à un fait internationalement illicite : rétorsion ou contre- mesure?
}

Emanuel Castellarin*

\begin{abstract}
Freezes of assets of foreign central banks are becoming common in the practice of the United States, Canada and the European Union as a reaction to breaches of international obligations by other States, currently including Syria and Iran. This article argues that they are wrongful in se, thus they must be qualified as counter-measures and that their wrongfulness can only be excluded under the conditions drawn by articles 49-53 of the ILC's draft articles on responsibility of States for internationally wrongful acts. Prima facie, the wrongfulness of freezes of assets of foreign central banks might stem from their inconsistency with the central banks' customary immunity of execution or with the articles of agreement of the IMF. In fact, they are inconsistent with the former but not with the latter.

State practice unanimously recognises immunity over the foreign central bank's assets used to enforce the foreign State's monetary policy, but is divided as to its extension to assets used for commercial activities. Notwithstanding the restrictive nature of the immunity, freezes of assets currently in force are not designed to comply with it, so that derogations do not cover all protected assets. On the other hand, freezes of assets of foreign central banks are consistent with monetary international law. True, they are restrictions to payments for current transactions, which are generally forbidden by article VIII sect. 2(a) of the IMF's articles of agreement. Nonetheless, they benefit from a tacit authorisation by the IMF's Executive Directors as they are not motivated by balance of payments concerns but by security reasons and have little impact on the international monetary system.
\end{abstract}

\footnotetext{
* Doctorant à l'École de Droit de la Sorbonne, Université Paris 1 Panthéon-Sorbonne ; Diplômé de l'Académie de droit international de la Haye (2012).
} 
E. Castellarin, « Le gel des avoirs d'une banque centrale étrangère comme réaction à un illicite : rétorsion ou contre-mesure ? », Annuaire de La Haye de droit international, vol. 25, 2012, pp. 173-197

\section{Introduction}

Le gel des avoirs ${ }^{1}$ d'une banque centrale étrangère ${ }^{2}$ se répand dans la pratique des Etats occidentaux et de l'Union européenne comme mesure de pression aux importants effets économiques en réaction à des conduites considérées comme internationalement illicites. Sa qualification alternative de rétorsion ou de contre-mesure, avec toutes les conséquences qui s'en suivent, impose de s'interroger sur le caractère licite ou illicite en soi de cette mesure.

Le fondement juridique du gel des avoirs peut être soit une résolution du Conseil de sécurité prise en vertu du Chapitre VII de la Charte, soit le droit international général, qui reconnaît comme licites tous les actes adoptés par un sujet dans l'exercice de ses compétences, à l'exclusion de ceux qui violent une règle internationale ${ }^{3}$. Puisque le gel des avoirs d'une banque centrale étrangère s'inscrit toujours dans une série de mesures restrictives discriminatoires, le contexte de son utilisation a changé dans le temps.

Pendant la guerre froide, le gel des avoirs des banques centrales étrangères fut sporadiquement couplé à celui des avoirs de leurs Etats. Le cas le plus connu, qui suivit les précédents concernant la Chine et Cuba, fut le gel américain des biens iraniens dans le cadre

\footnotetext{
${ }^{1}$ Le gel des avoirs est une mesure prise par un Etat rendant momentanément indisponibles les avoirs d'un étranger se trouvant sur le territoire de l'Etat ou entre les mains de ses ressortissants (J. Salmon (dir.), Dictionnaire de droit international public, Bruxelles, Bruylant, 2001 p. 529). Par «avoir» on entend ici tout bien ou crédit dont une personne peut entrer en possession ou détenir le contrôle.

${ }^{2}$ Il n'existe pas de définition juridique universelle du concept de «banque centrale », car celle-ci est établie par chaque Etat ou par traité (comme l'est la Banque Centrale Européenne). A défaut d'une définition juridique, elle ne peut être qualifiée que par les fonctions dont elle est investie par le ou les Etats qui l'ont créée, notamment par son privilège d'émission de la monnaie nationale, par la gestion des réserves officielles de change et par le rôle de prêteur de dernier ressort sur le plan national (J. Salmon, supra note 1, p. 121). La banque centrale exerce donc principalement des fonctions différentes de celles des banques commerciales, qui lui permettent la supervision et la régulation de la politique monétaire (Ch. Proctor, Mann on the Legal Aspects of Money, Oxford, Oxford University Press, $7^{\mathrm{e}}$ éd, 2012, pp. 570-571). Son degré d'indépendance n'est soumis à aucune règle de droit international (J. Salmon, ibid., p. 121).

${ }^{3}$ La licéité des actes non interdits peut avoir deux fondements alternatifs : une présomption de liberté en faveur des sujets internationaux ou une lacune du système juridique international. La présomption de liberté est classique (CPJI, arrêt du 7 septembre 1927, Lotus, série A n 10, p. 18) ; le raisonnement fondé sur une lacune de l'ordre juridique international, qui aboutit au même résultat, correspond à la métaphore de la traversée d'un carrefour autorisée non par un feu vert, mais par l'absence de feu rouge ( $v$. l'opinion individuelle du juge Guillaume à CIJ, avis consultatif du 8 juillet 1996, Licéité de la menace ou de l'emploi d'armes nucléaires, CIJ Rec. 1996, par. 9).
} 
E. Castellarin, « Le gel des avoirs d'une banque centrale étrangère comme réaction à un illicite : rétorsion ou contre-mesure ? », Annuaire de La Haye de droit international, vol. 25, 2012, pp. 173-197

de la crise des otages de Téhéran. ${ }^{4}$ Un autre gel des avoirs adopté par les Etas-Unis visa la Libye $^{5}$ et un gel d'avoirs réciproque fut adopté par le Royaume-Uni et par l'Argentine lors de la guerre des îles Falkland/Malouines en $1982 .{ }^{6}$ Toutes ces mesures étaient prises selon les pouvoirs d'urgence de l'exécutif dans le cadre d'un différend bilatéral. Après la fin de la guerre froide, le gel des avoirs des banques centrales est devenu un moyen de réaction décentralisée par plusieurs Etats à ce qu'ils considéraient comme un fait internationalement illicite $^{7}$ : les Etats-Unis s'en sont notamment servis contre 1'Iraq, le Soudan et la Birmanie/Myanmar, qui faisaient également l'objet de sanctions du Conseil de sécurité ${ }^{8}$ Tout en étant des mesures autonomes, ces gels des avoirs anticipaient ou prolongeaient donc des sanctions centralisées.

La résolution 1973 (2011) du Conseil de sécurité concernant la Libye marque 1'internationalisation de 1'emploi du gel des avoirs des banques centrales. ${ }^{9}$ En 2012, les avoirs de la Banque centrale d'Iran ont été gelés par l'Union européenne ${ }^{10}$ et par les Etats-Unis. ${ }^{11}$

\footnotetext{
${ }^{4}$ Executive Order 12170, 44 Fed. Reg. 65729, 14 novembre 1979; P. Juillard, Th. Flory, D. Carreau, « Chronique de droit internationale économique », AFDI, vol. 25 (1979), pp. 615-620.

${ }^{5}$ Executive Order 12544, 51 Fed. Reg. 1235, 8 janvier 1986.
}

${ }^{6}$ Control of Gold, Securities, Payments and Credits (Argentine Republic) Directions de 1982 (Statutory Instruments 1982, $\mathrm{n}^{\circ}$ 512), 3 avril 1982 ; A. F. Lowenfeld, International Economic Law, Oxford, Oxford University Press, 2008, p. 893.

${ }^{7}$ Sur le concept de réaction décentralisée $v$. F. Dopagne, Les contre-mesures des organisations internationales, Louvain-la-Neuve, Anthemis, 2010 ; D. Alland, Justice privée et ordre juridique international, Paris, Pedone, 1994 ; L. A. Sicilianos, Les réactions décentralisées à l'illicite, Paris, LGDJ, 1990.

${ }^{8}$ Dans le cas de l'Iraq, la mesure fut adoptée le 2 août 1990, avant que le Conseil de sécurité n'autorise l'adoption de sanctions contre l'invasion du Koweït: Executive Order 12722, 55 Fed. Reg. 31803 ; A. F. Lowenfeld, supra note 6, p. 871. Pour limiter les conséquences de l'invasion, les avoirs du Koweït, y compris de sa banque centrale, furent aussi saisis le même jour : Executive Order 12723, 55 Fed. Reg. 31805 ; H. L. Clark, K. A. Matthews, «Iraq/Kwait Economic Sanctions: Trade Relations on a War Footing », The International Lawyer, vol. 25 (1991), pp. 391-414.

${ }^{9} \mathrm{~S} / \mathrm{RES} / 1973$ (2011), par. 19. La résolution a été transposée dans l’UE par la décision 2011/137/PESC du Conseil du 28 février 2011, J.O. L 58, pp. 53-62 et par le règlement (UE) n 204/2011 du Conseil du 2 mars 2011, J.O. L 58, pp. 1-13. Les avoirs de la banque centrale ont ensuite été dégelés après la résolution 2009 (S/RES/2009 (2011), par. 15-19).

${ }^{10}$ Décision 2012/35/PESC du Conseil du 23 janvier 2012, J.O. L 19, pp. 22-30, art. 20 ; règlement (UE) $\mathrm{n}^{\circ}$ 267/2012 du Conseil du 23 mars 2012, J.O. L 88, p. 1-112, art. 23. 
E. Castellarin, « Le gel des avoirs d'une banque centrale étrangère comme réaction à un illicite : rétorsion ou contre-mesure ? », Annuaire de La Haye de droit international, vol. 25, 2012, pp. 173-197

L'UE $^{12}$ et le Canada ${ }^{13}$ ont aussi gelé les avoirs de la Banque centrale de Syrie. Dans la législation américaine, les banques centrales de Syrie, de Birmanie/Myanmar et du Soudan sont toujours comprises parmi les démembrements de l'Etat frappés par des gels d'avoirs. ${ }^{14}$

Le changement induit par la résolution 1973 (2011) est particulièrement important pour 1'Union européenne, ${ }^{15}$ qui avait déjà démontré dans les années 2000 être consciente de l'importance des banques centrales dans le financement d'activités internationalement illicites, ${ }^{16}$ mais n'avait jamais adopté de gel d'avoirs visant une banque centrale en tant que telle.

Dans le contexte actuel, le gel des avoirs d'une banque centrale a la fonction économique d'arrêter le financement d'activités considérées comme internationalement illicites, de manière directe s'il empêche l'acquisition des biens nécessaires à cette activité, ou de manière indirecte si la réaction choisie implique l'isolement de l'Etat ciblé du système financier international. En raison de la liberté étatique quant à l'organisation de sa banque

${ }^{11}$ National Defense Authorization Act for Fiscal Year 2012, H.R. $1540\left(112^{\text {th }}\right)$, section 1245, mise en œuvre par
un amendement de la Iranian Financial Sanctions Regulations (31 CFR 561) et par l'Executive Order 13599, 77 Fed. Reg. 6659, 5 février 2012.

${ }^{12}$ Décision 2012/739/PESC du 29 novembre 2012, abrogeant la décision 2011/782/PESC, J.O. L 330, pp. 21-51, art. 25 ; règlement (UE) $\mathrm{n}^{\circ} 36 / 2012$, art 14, J.O. L 16, p. 1-32, modifié par le Règlement (UE) $\mathrm{n}^{\circ} 168 / 2012 \mathrm{du}$ Conseil du 27 février 2012, J.O. L 54, pp. 1-5.

${ }^{13}$ Règlement sur les mesures économiques spéciales visant la Syrie, DORS/2011-114, 24 mai 2011, modifié le 5 mars 2012, Gazette du Canada, vol. 146, n 6, art. 3.

${ }^{14} 31$ CFR par. 537.307 (Birmanie/Myanmar); 31 CFR par. 538.305 (Soudan); 31 CFR par. 542.201 (Syrie), mis en œuvre par l'Executive Order 13582, 76 Fed. Reg. 52209, 17 août 2011.

${ }^{15}$ L’Union européenne détient une compétence exclusive pour l'adoption des mesures restrictives, dont la mise en œuvre incombe aux Etats membres (art. 215 TFUE; CJCE, 14 janvier 1997, The Queen, ex parte CentroCom v. HM Treasury and Bank of England, aff. C-124/95).

${ }^{16}$ Les gouverneurs des banques centrales, ainsi que certains de leurs collaborateurs, étaient visés par des gel de fonds individuels (Règlement $(\mathrm{CE}) \mathrm{n}^{\circ} 702 / 2008$ de la Commission du 23 juillet 2008 modifiant le règlement (CE) n $n^{\circ}$ 314/2004 du Conseil concernant certaines mesures restrictives à l'égard du Zimbabwe, J.O. L 195, pp. 19-21, annexe III ; Règlement (CE) n 353/2009 de la Commission du 28 avril 2009 modifiant le règlement (CE) $n^{\circ}$ 194/2008 du Conseil renouvelant et renforçant les mesures restrictives instituées à l'encontre de la Birmanie/du Myanmar, J.O. L 108, pp. 20-52, annexe I; Règlement (UE) $\mathrm{n}^{\circ}$ 1284/2009 du Conseil du 22 décembre 2009 instituant certaines mesures restrictives spécifiques à l'encontre de la République de Guinée, J.O. L 346, pp. 26-38, annexe II). Il était aussi déjà commun d'imposer des restrictions à l'activité des banques commerciales de l'Etat visé, ainsi que le gel des avoirs d'autres représentants de l'Etat, même jouissant d'une immunité internationale. 
E. Castellarin, « Le gel des avoirs d'une banque centrale étrangère comme réaction à un illicite : rétorsion ou contre-mesure ? », Annuaire de La Haye de droit international, vol. 25, 2012, pp. 173-197

centrale, certaines banques centrales sont contrôlées directement par le gouvernement, qui les utilise aussi pour l'accomplissent de fonctions typiques des banques commerciales, notamment la participation directe au commerce extérieur de l'Etat. Le gel des avoirs a des conséquences économiques particulièrement importantes pour certains Etats exportateurs de pétrole, comme la Libye et l'Iran. En effet, leurs banques centrales accomplissent toutes les fonctions typiques des banques centrales, mais participent aussi à l'allocation des ressources provenant du commerce du pétrole. ${ }^{17}$ Les banques centrales sont ciblées en raison de leur implication directe, dirigée par le gouvernement, dans les activités considérées comme internationalement illicites. ${ }^{18}$ Le gel de leurs avoirs s'inscrit donc dans le durcissement progressif des mesures restrictives à l'égard d'un certain Etat, qu'elles soient autorisées voire imposées par le Conseil de sécurité ou non. La Libye est le seul Etat dont la banque centrale a été ciblée par une résolution du Conseil de sécurité ; dans le cas de l’Iran, le gel des avoirs est

\footnotetext{
${ }^{17}$ M. H. Adeli, « The Consequences of Sanctions on Iran's Central Bank », Iranian Diplomacy, 4 février 2012, $<$ http://irdiplomacy.ir/en/page/1897602/The+Consequences + of + Sanctions + on + Iran $\%$ E2\%80\%99s + Central + Ban k.html>, consulté le 23 février 2013.

${ }^{18}$ Par exemple, dans le cas de la Libye, le Conseil de sécurité soutenait que la banque centrale était «[s]ous le contrôle de Mouammar Kadhafi et de sa famille, et source potentielle de financement de son régime»» (S/RES/1973 (2011), annexe II). Dans le cas de l'Iran, l’Union européenne considère qu' « il convient d'instituer des mesures restrictives à l'encontre de la Banque centrale d'Iran en raison de son implication dans des activités visant à contourner les sanctions infligées à l'Iran » (décision 2012/35/PESC, supra note 10, considérant 12) et les Etats-Unis que « [t] he financial sector of Iran, including the Central Bank of Iran, is designated as a primary money laundering concern ... because of the threat to government and financial institutions resulting from the illicit activities of the Government of Iran, including its pursuit of nuclear weapons, support for international terrorism, and efforts to deceive responsible financial institutions and evade sanctions » (National Defense Authorization Act for Fiscal Year 2012, supra note 11, section 1245, b)).
} 
E. Castellarin, « Le gel des avoirs d'une banque centrale étrangère comme réaction à un illicite : rétorsion ou contre-mesure ? », Annuaire de La Haye de droit international, vol. 25, 2012, pp. 173-197

une mesure autonome qui élargit les sanctions économiques du Conseil de sécurité $;{ }^{19}$ pour la Syrie, la mesure est totalement autonome. ${ }^{20}$

La question de la licéité du gel des avoirs des banques centrales étrangères en droit international est susceptible de se poser devant la CJUE et les juridictions de l'Etat qui exécute la mesure ${ }^{21}$, ou sur la scène internationale. ${ }^{22}$ Les mesures qui transposent une résolution du Conseil de sécurité ne soulèvent aucune question, sauf celle générale des limites des pouvoirs de cet organe ; les autres constituent les cas les plus sensibles politiquement, car ils reflètent la division du Conseil de sécurité quant à leur opportunité et la menace, voire l'utilisation, du droit de veto par l'un des membres permanents. De la licéité ou de l'illicéité intrinsèque de ces mesures autonomes dépend leur qualification comme rétorsions ou comme contre-mesures, qui déclenche des régimes juridiques distincts ${ }^{23}$ : alors que la rétorsion est un acte unilatéral inamical licite en lui-même, l'illicéité intrinsèque des contre-mesures est

${ }^{19}$ La résolution 1929 (S/RES/1929 (2010)), ne prévoit ni cette mesure ni un embargo sur les hydrocarbures, mais
dans le préambule elle note « le lien potentiel entre les recettes que l'Iran tire de son secteur de l'énergie et le
financement de ses activités nucléaires posant un risque de prolifération, et ... que le matériel et les matières
utilisés par les procédés chimiques de l'industrie pétrochimique sont très semblables à ceux qui sont employés
dans certaines activités sensibles du cycle du combustible nucléaire ». En parallèle au gel des avoirs de la banque
centrale, ont été adoptées des mesures autonomes concernant le commerce de ressources énergétiques, qui est
arrivé à compter pour 80 pour cent des exportations iraniennes, et d'autres domaines commerciaux et financiers.
${ }^{20}$ Aucune sanction économique n'a été adoptée par le Conseil de sécurité dans les résolutions sur les Moyen Orient concernant la Syrie : S/RES/2052 (2012); S/RES/2059 (2012); S/RES/2084 (2012); S/RES/2042 (2012) ; S/RES/2043 (2012).

${ }^{21}$ Il existe des précédents sur la licéité en droit américain de l'executive order de 1979 (supra note 4) : R. M. McGreevey, «The Iranian Crisis and US Law », Northwestern Journal of International Law \& Business, vol. 2 (1980), 383-454, pp. 400-422.

${ }^{22}$ A supposer qu'elle soit tenue au respect d'une certaine règle internationale, l'Union européenne, dotée de la personnalité internationale, peut engager sa responsabilité internationale, notamment si elle «donne des directives à un État ou à une autre organisation internationale et ... exerce un contrôle dans la commission du fait internationalement illicite » (CDI, Projet d'articles sur la responsabilité des organisations internationales, art. 15, <http://untreaty.un.org/ilc/texts/instruments/francais/commentaires/9_11_2011_francais.pdf $>$ ). Si l'Union n'engage pas sa responsabilité, ses Etats membres peuvent engager la leur, notamment «si, en se prévalant du fait que l'organisation est compétente relativement à l'objet d'une de [leurs] obligations internationales ..., il[s] contourne[nt] cette obligation en amenant l'organisation à commettre un fait qui, s'il avait été commis par [eux], aurait constitué une violation de cette obligation. » (art. 61, par. 1 du Projet d'articles précité). La responsabilité de l'Union et de ses Etats membres peut aussi être conjointe.

${ }^{23}$ G. Arangio-Ruiz, « $3^{\mathrm{e}}$ rapport sur la responsabilité des Etats », Annuaire de la CDI, 1991, vol. II, I, p. 10. 
E. Castellarin, « Le gel des avoirs d'une banque centrale étrangère comme réaction à un illicite : rétorsion ou contre-mesure ? », Annuaire de La Haye de droit international, vol. 25, 2012, pp. 173-197

exclue seulement si les Etats qui les adoptent et/ou l'Union européenne sont habilités à les adopter et si ces mesures respectent les conditions procédurales et substantielles codifiées aux articles 49-53 du projet d'articles de la Commission du droit international ("CDI") sur la responsabilité des Etats. ${ }^{24}$

S'appuyant sur le titre de compétence territorial ou personnel des Etats qui les adoptent, les gels des avoirs actuels ne suscitent pas les questions d'extraterritorialité qui ont contribué à rendre célèbre le gel des avoirs de la Banque centrale iranienne par les Etats-Unis de $1979 .{ }^{25}$ En outre, ils ne sont pas contraires à la Charte des Nations Unies. ${ }^{26}$ En revanche, on peut se poser la question de leur possible incompatibilité avec l'immunité coutumière des banques centrales, organes étatiques dont la protection internationale est nécessaire à l'exercice de la souveraineté économique d'un Etat (1). En outre, en raison de leur rôle, les gels d'avoirs pourraient empêcher les banques centrales d'accomplir les fonctions nécessaires à la participation des Etats au système monétaire international, dont la stabilité est protégée par les Statuts du FMI (2).

\footnotetext{
${ }^{24}$ Le projet d'articles sur la responsabilité des organisations internationales « ne traitent pas des conditions de licéité des contre-mesures lorsqu'elles sont prises par une organisation internationale lésée contre un État responsable »: CDI, Projet d'articles sur la responsabilité des organisations internationales, commentaire à 1'art. 22, par. 2 .

Pour une qualification de contre-mesure de l'ensemble des mesures restrictives de l'Union européenne contre l'Union, v. P.-E. Dupont, «Countermeasures and Collective Security: The Case of the EU Sanctions Against Iran », Journal of Conflict and Security Law, vol. 17 (2012), pp. 301-336, pp. 311-323. L'article soutient rapidement la thèse de l'illicéité du gel des avoirs de la banque centrale iranienne : p. 314.

${ }^{25}$ R. W. Edwards, « Extraterritorial Application of the U.S. Iranian Assets Control Regulations », AJIL, vol. 75 (1981), pp. 870-902 ; J. E. Hoffman, I. H. Giddy, «Lessons from the Iran Experience : National Currencies As International Money », Journal of Comparative Corporate Law and Securities Regulation, vol. 3 (1981), pp. 271-286.

${ }^{26}$ Les mesures restrictives autonomes par rapport aux résolutions du Conseil de sécurité ne sont pas eo ipso facto contraires à la Charte des Nations Unies, au moins si elles prolongent des résolutions du Conseil ( $v$. E. Lagrange, P. M. Eisemann, «Article 41 », in J.-P. Cot, A. Pellet, M. Forteau (dir.), La Charte des Nations Unies, Paris, Economica, $3^{\text {ème }}$ éd., 2005, pp. 1195-1242, p. 1204). Elles peuvent l'être si elles sont contraires à une résolution les interdisant ou imposant des mesures incompatibles, la primauté des obligations découlant de la Charte par rapport à d'éventuelles obligations conventionnelles contraires étant assurée par l'art. 103 de la Charte. Toutefois, ce n'est pas le cas en l'espèce.
} 
E. Castellarin, « Le gel des avoirs d'une banque centrale étrangère comme réaction à un illicite : rétorsion ou contre-mesure ? », Annuaire de La Haye de droit international, vol. 25, 2012, pp. 173-197

1. La compatibilité du gel des avoirs avec l'immunité d'exécution des banques centrales

Dans l'affaire des immunités juridictionnelles Allemagne c. Italie, la Cour internationale de justice ("CIJ") a eu l'occasion de rappeler que la règle coutumière de l'immunité des Etats, «solidement enracinée dans la pratique contemporaine des Etats», ${ }^{27}$ « procède du principe de l'égalité souveraine des Etats qui, ainsi que cela ressort clairement du paragraphe 1 de l'article 2 de la Charte des Nations Unies, est l'un des principes fondamentaux de l'ordre juridique international $»{ }^{28}$ Ainsi, l'Etat jouit d'une immunité d'exécution à l'étranger, ${ }^{29}$ qui opère comme une exception en droit procédural interne, mais aussi comme une règle substantielle dont la violation est internationalement illicite. A la différence de l'immunité de juridiction, qui permet à l'Etat d'échapper à la compétence des juridictions étrangères, l'immunité d'exécution lui permet d'échapper aux mesures de contrainte ou d'exécution forcée de la part d'un autre Etat. ${ }^{30}$ Le débat sur l'immunité d'exécution a toujours été centré sur son côté juridictionnel; or les mesures restrictives, adoptées par le pouvoir législatif et mises en œuvre par le pouvoir exécutif, sont extrajuridictionnelles. Si le pouvoir judiciaire étatique n'est pas appelé par la banque centrale étrangère à faire primer l'immunité coutumière sur le gel des avoirs, il n'aura pas l'occasion de se prononcer sur la question. Les précédents sont très rares : la compatibilité du gel américain avec l'immunité de la banque centrale iranienne s'est notamment posée entre 1979 et 1980 en relation aux engagements internationaux des Etats-Unis envers l'Iran, qui dépendent aussi bien de la portée de l'immunité accordée par la législation nationale que de son articulation avec le traité d'amitié de 1955 entre les deux Etats. ${ }^{31}$ Dans quelle mesure

\footnotetext{
${ }^{27}$ CDI, ACDI, 1980, vol. II, II, p. 144 ; CIJ, Immunités juridictionnelles de l'État (Allemagne c. Italie ; Grèce (intervenant)), arrêt du 3 février, par. 56, <http://www.icj-cij.org/docket/files/143/16884.pdf>.

${ }^{28} \mathrm{CIJ}$, ibid., par. 57.

${ }^{29}$ H. Fox, The Law of State Immunity, Oxford, Oxford University Press, $2^{\mathrm{e}}$ éd., 2008, pp. 464-473 ; A. Dickinson, R. Lindsay, J. P. Loonam, State Immunity. Selected Materials and Commentary, Oxford, Oxford University Press, 2004 ; L. G. Radicati di Borzolo, La giurisdizione esecutiva e cautelare nei confronti degli Stati stranieri, Milano, Giuffrè, 1992, pp. 180-186 ; C. H. Schreuer, State immunity : some recent developments, Cambridge, Grotius, 1988, pp. 156-159 ; A. Reinisch, «European Court Practice Concerning State Immunity from Enforcement Measures », EJIL, vol. 17 (2006), 803-836, pp. 826-827; L. Gramlich, "Staatliche Immunität für Zentralbanken? », Rabels Zeitschrift, vol. 45 (1981), pp. 545-601.

${ }^{30} \mathrm{~J}$. Salmon, supra note 1, pp. 559-560.

${ }^{31}$ R. M. McGreevey, supra note 21.
} 
E. Castellarin, « Le gel des avoirs d'une banque centrale étrangère comme réaction à un illicite : rétorsion ou contre-mesure ? », Annuaire de La Haye de droit international, vol. 25, 2012, pp. 173-197

peut-on appliquer aux mesures restrictives visant des banques centrales étrangères des règles conçues pour être invoquées devant les juridictions internes? On ne peut pas exclure le développement d'un droit coutumier autonome des saisies extra-juridictionnelles, mais, en l'absence d'une pratique établie en la matière, les règles sur l'immunité d'exécution juridictionnelle s'imposent par analogie : en effet, du point de vue du droit international l'Etat qui gèle les avoirs est responsable quel que soit l'organe qui adopte ou met en ouvre la mesure. $^{32}$

Les Etats se partagent entre la conception absolue et la conception relative de l'immunité : alors que la première reconnaît aux biens de la structure étatique une protection inconditionnée liée à sa nature souveraine, la seconde est fondée sur la protection fonctionnelle des seuls biens que l'Etat affecte à ses activités souveraines, à l'exclusion de ses activités commerciales. ${ }^{33}$ La renonciation à une éventuelle immunité, juridiquement possible, est pratiquement inconcevable lorsque le gel concerne une partie importante des avoirs d'une banque centrale. La pratique étatique, codifiée par les textes internationaux, a reconnu aux banques centrales une immunité, dont il est raisonnable de croire qu'elle est relative (1.1). Malgré le caractère restrictif de cette immunité, les gels des avoirs actuellement en vigueur ne sont pas totalement compatibles avec elle (1.2).

\subsection{La nature relative de l'immunité}

La division de la pratique étatique en matière d'immunité d'exécution des banques centrales (1.1.1) se reflète dans la difficulté d'identifier la règle coutumière, qui concerne donc seulement les avoirs affectés à des activités accomplies jure imperii (1.1.2).

\subsubsection{Le partage des pratiques étatiques}

Traditionnellement, la pratique étatique majoritaire en matière d'immunité ne distinguait pas les banques centrales des autres entités étatiques. Cette pratique influence encore une partie des Etats dotés d'une législation en matière d'immunités des Etats étrangers,

\footnotetext{
${ }^{32} \mathrm{CDI}$, «Projet d'articles sur la responsabilité de l'Etat pour fait internationalement illicite », $A C D I, 2001$, vol. II, II, p. 26, art. 4.

${ }^{33}$ v. E. K. Bankas, The State Immunity Controversy in International Law, Berlin, Springer, 2005.
} 
E. Castellarin, « Le gel des avoirs d'une banque centrale étrangère comme réaction à un illicite : rétorsion ou contre-mesure ? », Annuaire de La Haye de droit international, vol. 25, 2012, pp. 173-197

notamment l'Australie, ${ }^{34}$ et une bonne partie des Etats où le régime des immunités est jurisprudentiel, comme l'Allemagne. ${ }^{35}$ Dans les années 1970, les législations britannique et américaine sur les immunités souveraines ont introduit des dispositions spécifiques sur l'immunité d'exécution des biens des banques centrales, qui ont clarifié définitivement que la banque centrale est un organe étatique. En effet, avant l'adoption de ces législations, certains Etats, influencés par la doctrine classique de l'immunité absolue, ne reconnaissaient l'immunité qu'aux démembrements de l'Etat dépourvus de la personnalité morale, excluant ainsi la plupart des banques centrales. ${ }^{36}$

Les tentatives de codification internationale confirment le dépassement de la vielle approche structurelle, car à l'instar des législations britannique et américaine, elles prévoient toujours une disposition spéciale sur les biens des banques centrales. ${ }^{37}$ Les banques centrales jouissent donc certainement d'une immunité coutumière, mais afin d'établir si tout gel de leurs avoirs est illicite il faut déterminer si tous les avoirs ou si seuls les avoirs affectés à une activité souveraine sont protégés par l'immunité. La pratique de certains Etats reste inspirée par la conception absolue de l'immunité, alors que d'autres sont inspirées par la conception relative et fonctionnelle : les Etats particulièrement intéressés à cause de la présence sur leur territoire d'importants centres financiers se partagent entre les deux camps.

La législation britannique, imitée par certains Etats de tradition de common law, ${ }^{38}$ accorde aux banques centrales étrangères une immunité d'exécution large, dont la doctrine

\footnotetext{
${ }^{34}$ Foreign States Immunity Act, Act No. 196 of 1985, section 35(1).

${ }^{35}$ H. Fox, supra note 29, p. 472 ; Ch. Steven, B. Krauskopf, « Immunity of foreign central banks under German law », Journal of International Financial Markets, vol. 2 (2000), pp. 138-149.

${ }^{36}$ Trendtex Trading Corporation c. Central Bank of Nigeria, All England Law Report 1977, vol. 1, pp. 881-882.

${ }^{37}$ La doctrine avait suggéré que les législations britannique et américaine, qui ont fait ce choix pour la première foi, comprenaient une disposition spéciale sur les banques non pour se conformer à une opinio juris, mais comme conséquence d'un choix politique favorable à la permanence des banques centrales étrangères sur les places financières de Londres et New York (W. Blair, « The Legal Status of Central Bank Investments under English Law », Cambridge Law Journal, vol. 57 (1998), pp.374-390, p. 378 ; J. R. Crawford, « International Law and Foreign Sovereigns : Distinguishing Immune Transactions », British Yearbook of International Law, vol. 54 (1983), pp. 75-118, p. 118). Cette hypothèse a été avancée aussi pour la législation chinoise de 2005, inspirée par les nécessités de Hong Kong et par le souhait de favoriser aussi Macao (L. Zhu, «State Immunity from Measures of Constraint for the Property of Foreign Central Banks : the Chinese Perspective », Chinese Journal of International Law, vol. 6 (2007), pp. 67-81).

${ }^{38}$ Singapore, Sovereign Immunity Act de 1979, art. 16 par. 4 ; Pakistan, State Immunity Ordinance de 1981, art. 15, par. 4 ; Afrique du Sud, Foreign States Immunities Act de 1981, art. 15, par. 3.
} 
E. Castellarin, « Le gel des avoirs d'une banque centrale étrangère comme réaction à un illicite : rétorsion ou contre-mesure ? », Annuaire de La Haye de droit international, vol. 25, 2012, pp. 173-197

considère qu'elle interdit la saisie des avoirs indépendamment de leur affectation. ${ }^{39} \mathrm{La}$ République Populaire de Chine, fidèle à sa conception absolue de l'immunité, considère aussi l'immunité d'exécution des banques centrales étrangères comme indépendante de l'affectation des biens. ${ }^{40}$ En l'absence de renonciation à l'immunité, qu'elles permettent, ces législations ont l'avantage de protéger le consentement de la banque centrale à la saisie de ses biens, car elles empêchent que l'Etat qui l'opère distingue lui-même les actes jure imperii des actes jure gestionis. En France, malgré la nature principalement jurisprudentielle du droit des immunités, le législateur a consacré dans l'article L. 153-1, al. $1^{\text {er }}$ du Code monétaire et financier ("CMF") une approche particulièrement protectrice des biens des banques centrales : «[n]e peuvent être saisis les biens de toute nature, notamment les avoirs de réserves de change, que les banques centrales ou les autorités monétaires étrangères détiennent ou gèrent pour leur compte ou celui de l'Etat ou des Etats étrangers dont elles relèvent », avec la seule exception, prévue à l'alinéa 2 , des biens faisant partie «d'un patrimoine qu[e la banque centrale] affecte à une activité principale relevant du droit privé ${ }^{4} .{ }^{41}$

Dans la pratique d'autres Etats, l'immunité des biens des banques centrales est relative. Ainsi, selon le Foreign Sovereign Immunities Act américain de 1976,

« the property of a foreign state shall be immune from attachment and from execution, if ... (1) the property is that of a foreign central bank or monetary authority held for its own account, unless such bank or authority, or its parent foreign government, has explicitly waived its immunity from attachment in aid of execution, or from execution, notwithstanding any withdrawal of the waiver which the bank, authority or government may purport to effect except in accordance with the terms of the waiver $\gg{ }^{42}$

\footnotetext{
${ }^{39}$ State Immunity Act de 1978, section 14(4) : «Property of a State's central bank or other monetary authority shall not be regarded ... as in use or intended for use for commercial purposes; and where any such bank or authority is a separate entity [procedural privileges] shall apply to it as if references to a State were references to the bank or authority. »; R. Higgins, «Execution of State Property : United Kingdom Practice », Netherlands Yearbook of International Law, vol. 10 (1979), pp. 35-54, p. 51 ; H. Fox, «Enforcement Jurisdiction, Foreign State Property and Diplomatic Immunity », ICLQ, vol. 34 (1985), pp. 115-141, p. 137.

${ }^{40}$ Loi sur l'immunité juridictionnelle d'exécution des biens des banques centrales étrangères de 2005, art.1, reproduit in L. Zhu, supra note 37 : la législation chinoise démontre que, si l'Etat du for se refuse de distinguer les deux catégories de biens, la renonciation à l'immunité par l'Etat protégé joue un rôle fondamental.

${ }^{41}$ Cet alinéa s'applique seulement si le demandeur dispose d'un titre exécutoire, introduisant ainsi une différence de régime entre la saisie conservatoire et la saisie-exécution, qui caractérise aussi la jurisprudence américaine $(\mathrm{H}$. Fox, supra note 29, p. 471).

${ }^{42}$ USC 28 par. 1611 lett. b.
} 
E. Castellarin, « Le gel des avoirs d'une banque centrale étrangère comme réaction à un illicite : rétorsion ou contre-mesure ? », Annuaire de La Haye de droit international, vol. 25, 2012, pp. 173-197

L'expression «for its own account» a été interprété par la doctrine et les juridictions américaines comme se référant aux seuls biens affectés à des activités jure imperii. ${ }^{43}$ Le Canada a adopté une législation similaire, subordonnant clairement l'immunité à l'affectation des biens à une activité non commerciale. ${ }^{44}$ Plusieurs Etats de tradition romano-germanique appliquent pour les biens des banques centrales le critère de l'affectation, comme pour les biens des autres organes étatiques. Ainsi, les biens affectés à une activité souveraine ne peuvent pas être saisis, ${ }^{45}$ mais ceux affectés à une activité commerciale peuvent l'être. ${ }^{46}$ Par activité jure imperii il faut entendre, dans le cas des banques centrales, leurs activités typiques de gestion directe de la politique monétaire, à l'exclusion de celles qui sont normalement accomplies par des banques commerciales, comme le financement et la distribution des bénéfices d'activités commerciales. ${ }^{47}$

Aussi bien la conception absolue que la conception relative de l'immunité comportent un risque d'abus, c'est-à-dire d'utilisation de droits détournée de leur but et dommageable à d'autres sujets. ${ }^{48}$ L'Etat qui bénéficie d'une immunité absolue peut l'utiliser pour fausser la concurrence avec des opérateurs commerciaux privés; l'Etat qui ne reconnaît qu'une immunité relative peut qualifier d'affectés à des activités commerciales des biens que d'autres considéreraient comme affectés à des activités souveraines. En effet, un problème crucial est celui de la détermination de l'affectation des biens à une certaine activité : elle est facilitée par l'appartenance, rare en pratique, des biens à un patrimoine distinct affecté à une certaine activité, mais elle peut être très délicate si tous les biens de la banque centrale font partie d'un

\footnotetext{
${ }^{43}$ E. T. Patrikis, «Central Bank Property: Immunity from attachment in the United States », University of Illinois Law Review, 1982, p. 265-287, p. 277 ; Banque Campafina c. Banco de Guatemala, 599 F Supp. 329 (SDNY 1984), ILR, vol. 92, pp. 399-405, p. 402.

44 «Sous réserve [de renonciation], sont insaisissables les biens qu'une banque centrale ou une autorité monétaire étrangères détiennent pour leur propre compte et qui ne sont pas utilisés ou destinés à être utilisés dans le cadre d'une activité commerciale » (loi sur l'immunité des États, L.R.C. (1985), ch. S-18), section 12 (4).

${ }^{45}$ P. ex. Cass. civ. 1ère 3 novembre 1952, Epoux Martin c. Banque d'Espagne ; Cass. civ. $1^{\text {re }}, 19$ mai 1976 , Zavicha Blagojevic c. Banque du Japon.

${ }^{46}$ Landgericht Frankfurt, Y.M.N. Establishment c. Central Bank of Nigeria, 2 décembre 1975, ILR, vol. 65, p. 131 et seq., p. 137 ; Cour d'appel de Brussels, Leica AG c. Central Bank of Iraq et Etat irakien, 15 février 2000, Journal des tribunaux (Belgique), n 6 (2001); Tribunal fédéral suisse, Banque centrale de la République de Turquie c. Weston Compagnie de Finance et d'Investissement SA, 15 novembre 1978, ILR, vol. 65, p. 417 et seq.

${ }^{47}$ Supra note 2.

${ }^{48}$ v. A. Ch. Kiss, L'abus de droit en droit international, Paris, LGDJ, 1953.
} 
E. Castellarin, « Le gel des avoirs d'une banque centrale étrangère comme réaction à un illicite : rétorsion ou contre-mesure ? », Annuaire de La Haye de droit international, vol. 25, 2012, pp. 173-197

même patrimoine affecte à la fois à des activités jure gestionis et à des activités jure imperii, comme c'est le cas normalement. ${ }^{49}$ Dans cette hypothèse, l'affectation des biens à l'une ou à l'autre n'est que potentielle: les législations et les jurisprudences défendant l'immunité relative ne peuvent qu'introduire une présomption d'affectation, faisant peser sur la partie qui soutient le contraire la charge de la preuve.

Par exemple, la jurisprudence suisse ${ }^{50}$ ne reconnaît pas d'immunité à des comptes de banques centrales étrangères à défaut de preuve qu'ils sont utilisés pour des activités souveraines ; la jurisprudence française antérieure à l'introduction du code L.153-1 du CMF en 2005 retenait le même raisonnement. ${ }^{51} \mathrm{Au}$ contraire, une juridiction fédérale américaine a renoncé à saisir certains fonds privés pourtant saisissables détenus par une banque centrale étrangère car ils étaient impossibles à distinguer de ceux utilisés pour des activités souveraines. ${ }^{52}$ Un exemple de la difficulté de l'établissement de la présomption est fourni par la jurisprudence allemande: alors que dans une affaire un Landgericht a conclu que l'éventualité de l'affectation des biens a une activité souveraine ne suffisait pas à activer l'immunité, ${ }^{53}$ dans une autre affaire des bénéfices du commerce de pétrole ont été considérés par le Bundesverfassungsgericht comme protégés par l'immunité une fois entrés dans le compte d'une banque centrale affectée à des activités souveraines, indépendamment de leur utilisation finale. ${ }^{54}$ En effet, cette difficulté est particulièrement aiguë lorsque le gel des comptes bancaires de la banque centrale présente un intérêt réel car les bénéfices du commerce des hydrocarbures peuvent y être versés, comme dans le cas de l'Iran.

1.1.2 L'existence d'une immunité coutumière pour les seuls avoirs affectés à une activité souveraine

\footnotetext{
49 J. R. Crawford, «Execution of Judgments and Foreign Sovereign Immunity, » AJIL, vol. 75 (1981), 820-869, 865

${ }^{50}$ Tribunal fédéral suisse, République socialiste du peuple arabe de Lybie-Jamahiriya c. Actimon SA, 24 Avril 1985, ILR vol. 82, p. 30 et seq., p. 35.

${ }^{51}$ Cass. civ. $1^{\text {ère }}, 11$ février 1969, Englander c. Statni Banka Ceskoslovenska.

${ }^{52}$ Weston Compagnie de Finance et d'Investissement v. La Republica del Ecuador, 823 F Supp. 1106 (SDNY 1993).

${ }^{53}$ Supra note 46.

${ }^{54}$ Bundesverfassungsgericht, National Iranian Oil Company, Recueil BVerfGE, aff. 678, 679, 680, 681, 683/81, vol. 64, p. 1.
} 
E. Castellarin, « Le gel des avoirs d'une banque centrale étrangère comme réaction à un illicite : rétorsion ou contre-mesure ? », Annuaire de La Haye de droit international, vol. 25, 2012, pp. 173-197

Comme la pratique des Etats, les tentatives de codification en la matière ont aussi adopté des points de vue différents : l'Institut de Droit International ("IDI") et l'International Law Association ("ILA") ont défendu l'immunité relative, alors que la CDI a choisi une formulation plus générale. La résolution de l'IDI sur les aspects récents de l'immunité de juridiction et d'exécution des Etats, adoptée à Bâle en 1991 prévoit que «les catégories suivantes de biens d'un Etat bénéficient de l'immunité d'exécution ... c) les biens de la Banque centrale ou de l'autorité monétaire de l'Etat utilisés pour leurs besoins propres ou dont l'utilisation à ces fins est prévue $\gg .{ }^{55}$ Le projet d'articles de l'ILA révisé à Buenos Aires en 1994 est également restrictif : «Attachment or execution shall not be permetted, if ... 3. The property is that of a State central bank held by it for central banking purposes ». ${ }^{56}$

La Convention des Nations Unies sur l'immunité juridictionnelle des Etats et de leurs biens du 2 décembre 2004, ${ }^{57}$ issue d'un projet d'articles de la CDI, est considérée comme la tentative de codification la plus significative en la matière. Selon son article 19 (Immunité des États à l'égard des mesures de contrainte postérieures au jugement),

« [a]ucune mesure de contrainte postérieure au jugement, telle que saisie, saisie-arrêt ou saisie-exécution, ne peut être prise contre des biens d'un État en relation avec une procédure intentée devant un tribunal d'un autre État excepté si et dans la mesure où : ...c) Il a été établi que les biens sont spécifiquement utilisés ou destinés à être utilisés par l'État autrement qu'à des fins de service public non commerciales et sont situés sur le territoire de l'État du for, à condition que les mesures de contrainte postérieures au jugement ne portent que sur des biens qui ont un lien avec l'entité contre laquelle la procédure a été intentée. »

A ce propos, l'article 21 (Catégories spécifiques de biens) prévoit explicitement au par. 1 que «[1]es catégories de biens d'État ci-après ne sont notamment pas considérées comme des biens spécifiquement utilisés ou destinés à être utilisés par l'État autrement qu'à des fins de service public non commerciales au sens des dispositions de l'alinéa $c$ de l'article 19 : ...c) Les biens de la banque centrale ou d'une autre autorité monétaire de l'État ». Le sens de cet article est clair : il n'est pas raisonnable d'interpréter cette disposition comme

\footnotetext{
${ }^{55}$ Résolution de l'IDI, Les aspects récents de l'immunité de juridiction et d'exécution des Etats, session de Bâle, 1991, art. 4, par. 2 lett. c.

${ }^{56}$ ILA, Revised Draft Articles for a Convention on State Immunity, Buenos Aires, 1994, art. VIII lett. C.3, 4.

${ }^{57}$ G. Hafner, L. Lange, « La Convention des Nations Unies sur l'immunité juridictionnelle des Etats et de leurs biens », AFDI, vol. 50 (2004), pp. 45-76.
} 
E. Castellarin, « Le gel des avoirs d'une banque centrale étrangère comme réaction à un illicite : rétorsion ou contre-mesure ? », Annuaire de La Haye de droit international, vol. 25, 2012, pp. 173-197

comprenant implicitement une exception pour les biens utilisés pour des activités commerciales. L'exclusion expresse par la CDI, faute d'un appui suffisant, des mots proposés par le Rapporteur spécial « et utilisés à des fins monétaires » à la fin du texte ${ }^{58}$ confirme le sens ordinaire des termes. La portée de cette disposition est clarifiée par les commentaires de la CDI à son projet d'articles. A priori, on pourrait se demander si la présomption qu'elle institue est réfragable ou irréfragable : le terme «considérées » pourrait indiquer tant une orientation pour l'interprète qu'une véritable fiction juridique. En d'autres termes, le juge de l'exécution, ou par analogie l'autorité chargée du gel des avoirs, doit-il se contenter de considérer tous les biens de la banque centrale comme utilisés seulement à des fins de service public non commerciales ou peut-il se référer à l'utilisation réelle des biens, considérant cette disposition seulement comme un cas de figure de la maxime in dubio mitius ? La CDI précise que cette disposition «vise à protéger certaines catégories de biens, en les excluant de toute présomption de consentement à des mesures de contrainte ${ }^{59}$ et entend réagir à la jurisprudence des juridictions nationales permettant la saisie des biens des banques centrales étrangères. ${ }^{60}$

La valeur de cet article est pourtant douteuse : reflète-t-il le droit coutumier? La Convention n'est pas encore en vigueur, faute d'un nombre de ratifications suffisantes ${ }^{61}$ et l'approche délibérément "militante" du projet d'articles de la CDI, qui réagit aux jurisprudences nationales permettant la saisie, fait état d'une intention de développement du droit international plutôt que de sa codification. En effet, la codification internationale ne peut pas recomposer une pratique étatique divisée sur la portée de l'immunité des biens des banques centrales. Dans ces conditions, le droit coutumier se forme a minima, mais, devant présumer la limitation d'une souveraineté, s'agit-il de celle de l'Etat qui adopte la saisie ou de celle de l'Etat dont les biens sont saisis ? La règle de l'immunité d'exécution peut opérer sur certains biens de la banque centrale mais pas sur d'autres sans qu'il existe des doutes sur son

\footnotetext{
${ }^{58}$ CDI, « Projet d'articles sur les immunités juridictionnelles des Etats et de leurs biens », ACDI, 1991, vol. II, II, p. 62, Commentaire à l'art 19 par. 1, par. 5 .

${ }^{59}$ Ibid., p. 61, par. 1.

${ }^{60}$ Ibid., par. 2.

${ }^{61}$ La Convention été signée par 28 Etats, et ratifiée par 13 sur les 30 nécessaires pour l'entrée en vigueur (art. $30)$.
} 
E. Castellarin, « Le gel des avoirs d'une banque centrale étrangère comme réaction à un illicite : rétorsion ou contre-mesure ? », Annuaire de La Haye de droit international, vol. 25, 2012, pp. 173-197

contenu, qui est l'interdiction de rendre indisponibles les biens. ${ }^{62} \mathrm{Il}$ serait déraisonnable de conclure qu'aucun bien des banques centrales étrangères n'est protégé par une immunité : la division de la pratique étatique sur les biens affectés à des activités commerciales ne remet pas en question l'existence de l'immunité pour les biens considérés par l'autorité qui décide la saisie comme utilisés pour des activités souveraines. Le droit coutumier positif contient donc uniquement une immunité relative.

Le gel des avoirs des banques centrales est donc licite si les avoirs sont affectés à une activité jure gestionis, comme le commerce d'hydrocarbures. Le but essentiel de la mesure peut donc être atteint de manière licite en droit international, à condition de respecter l'immunité des avoirs affectés à des activités jure imperii; or ce n'est pas le cas des gels des avoirs actuellement en vigueur.

1.2 L'incompatibilité partielle des gels des avoirs en vigueur avec l'immunité

Malgré les dérogations prévues (1.2.1), les gels d'avoirs révèlent une inspiration qui les empêche de respecter l'immunité des avoirs affectés à des activités souveraines (1.2.2).

\subsubsection{Des dérogations indépendantes de l'affectation des avoirs}

S'ils s'étendaient à tous les avoirs des banques centrales ciblées, les gels des avoirs des banques centrales étrangères en vigueur seraient clairement incompatibles avec l'immunité de certains d'entre eux. Or ils prévoient toujours des dérogations octroyées par l'exécutif au cas par cas, qui toutefois ne couvrent pas tous les avoirs protégés par l'immunité. En effet, l'immunité relative est inspirée par la nécessite de satisfaire les droits des créditeurs en traitant de la même manière tous les opérateurs économiques qui accomplissent le même type d'activité commerciale. ${ }^{63}$ Or la finalité du gel des avoirs des banques centrales n'est pas

\footnotetext{
${ }^{62}$ Cette situation se distingue de celle où le contenu même de la règle est défini par une pratique étatique non uniforme, par exemple celle du droit d'asile en Amérique latine dans l'affaire Haya de la Torre (CIJ, Droit d'asile (Colombie c. Pérou), arrêt du 20 novembre 1950, Recueil 1950, p. 277).

${ }^{63}$ I. Pingel-Lenuzza, Les immunités des Etats en droit international, Bruxelles, Bruylant, 1998, pp. 46-53. Pour un plaidoyer récent pour la nécessité d'élargir les conséquences de cette ratio legis, concernant les comptes bancaires des ambassades mais valable aussi pour ceux des banques centrales étrangères, v. C. Ryngaert, «Embassy Bank Accounts and State Immunity from Execution: Doing Justice to the Financial Interests of Creditors », Leiden Journal of International law, vol. 26 (2013), pp. 73-88.
} 
E. Castellarin, « Le gel des avoirs d'une banque centrale étrangère comme réaction à un illicite : rétorsion ou contre-mesure ? », Annuaire de La Haye de droit international, vol. 25, 2012, pp. 173-197

la régulation des phénomènes économiques, mais l'influence sur la conduite d'un autre Etat. Les dérogations ne sont pas dictées par le respect du souverain étranger mais par des préoccupations économiques et humanitaires en faveur de la population, comme le démontre le Conseil de l'Union européenne pour l'Iran :

«compte tenu de l'implication possible de cette dernière dans le financement du commerce extérieur, des dérogations sont jugées nécessaires afin que cette mesure financière ciblée n'empêche pas les opérations commerciales, y compris les contrats portant sur des vivres, des soins de santé, des équipements médicaux ou répondant à des besoins humanitaires $»{ }^{64}$

A cause de cette différente inspiration, certains avoirs affectes à des activités souveraines peuvent faire l'objet d'un gel, qui est alors incompatible avec l'immunité qui les protège.

Aux Etats-Unis les dérogations aux gels des avoirs des banques centrales sont régies en général par la partie 501 du chapitre 31 du Code of Federal Regulations, qui est peu adapté à éviter la violation de l'immunité protégeant les avoirs affectés à des activités souveraines. Des règles plus détaillées concernent les mesures restrictives concernant l'Iran, au terme desquelles elles

« shall apply with respect to a foreign financial institution owned or controlled by the government of a foreign country, including a central bank of a foreign country, only insofar as it engages in a financial transaction for the sale or purchase of petroleum or petroleum products to or from Iran conducted or facilitated on or after that date that is 180 days after the date of the enactment of this Act. ${ }^{65}$

De manière similaire, les décisions du Conseil n'interdisent pas « un paiement par une institution financière non désignée dû au titre d'un contrat commercial spécifique, dès lors que l'État membre concerné a établi, au cas par cas, que le paiement n'est pas reçu directement ou indirectement par une personne ou entité visée [par d'autres mesures] », les transferts ayant «pour objet de fournir aux institutions financières relevant de la juridiction des États membres des liquidités en vue du financement d'échanges commerciaux, dès lors que le transfert a été autorisé par l'État membre concerné » et les "paiements à la Banque centrale d'Iran versés en exécution d'obligations [non relatives aux biens et services soumis à

\footnotetext{
${ }^{64}$ Règlement 267/2012, supra note 10 , considérant 12.

${ }^{65}$ National Defense Authorization Act for Fiscal Year 2012, supra note 11, section 1245, lett. d, par. 3.
} 
E. Castellarin, « Le gel des avoirs d'une banque centrale étrangère comme réaction à un illicite : rétorsion ou contre-mesure ? », Annuaire de La Haye de droit international, vol. 25, 2012, pp. 173-197

embargo] $\gg{ }^{66}$ Les conditions de fond et de procédure de ces dérogations, qui incombent aux Etats membres, sont précisées par les règlements. ${ }^{67}$

\subsubsection{La protection insuffisante des avoirs couverts par l'immunité}

Puisque les avoirs qui font l'objet d'une dérogation sont identifiés seulement par le secteur commercial de provenance ou d'affectation, aucune référence n'est faite à leur affectation à des activités jure imperii. Les Etats-Unis et l'Union européenne n'ont donc pas pris en compte l'immunité d'exécution des banques centrales étrangères, alors que le Conseil de l'Union européenne a prévu des dérogations pour les avoirs nécessaires aux activités officielles de missions diplomatiques ou consulaires ou d'organisations internationales. ${ }^{68}$ Les dérogations aux gels des avoirs peuvent couvrir aussi bien des transferts vers la banque centrale que des paiements effectués par la banque centrale. Parmi les avoirs qui entrent dans le patrimoine de la banque centrale, certains peuvent être débloqués en raison de leur provenance, non liée à un secteur commercial ciblé par les mesures restrictives. La banque centrale peut affecter ces avoirs à une activité jure imperii ou jure gestionis, pourvu que cette dernière ne soit pas ciblée par les mesures restrictives. Les autres avoirs, provenant d'un secteur ciblé par les mesures restrictives, sont gelés sans égard à leur affectation future. ${ }^{69}$

Le gel sélectif des avoirs ne poserait pas problème si leur déblocage était possible pour financer toutes les activités jure imperii de la banque centrale. Or le déblocage des avoirs est possible seulement pour financer des activités commerciales, par ailleurs non ciblées par les mesures restrictives. Les transferts ainsi autorisés sont possibles seulement au sein de l'Union européenne, à l'exclusion donc du transfert vers un Etat tiers et du rapatriement des avoirs. De plus, les dérogations sont à interpréter de manière restrictive pour éviter tout contournement ${ }^{70}$.

\footnotetext{
${ }^{66}$ Décision 2012/35/PESC, supra note 10, art. 21, par. 7-9

${ }^{67}$ Règlement 267/2012 (Iran), supra note 10, art. 28 ; règlement 36/2012 (Syrie), supra note 12, art. 21 bis, introduit par le Règlement (UE) n ${ }^{\circ}$ 867/2012 du Conseil du 24 septembre 2012, J.O. L 257, pp. 1-2.

${ }^{68}$ Règlement 267/2012 (Iran), supra note 10, art. 27 ; règlement 36/2012 (Syrie), supra note 12, art. 16.

${ }^{69}$ Lors qu'il est certain que des biens sont affectés à la satisfaction d'une demande judiciaire ou arbitrale, il est pourtant logique et compatible avec l'immunité de les débloquer (Règlement 267/2012 (Iran), supra note 10, art. 24, lett. a).

${ }^{70}$ En particulier, le Conseil de l'UE prévoit dans le cas de l'Iran: « Eu égard aux tentatives de l'Iran d'utiliser son système financier pour contourner les sanctions, il est nécessaire d'exiger une vigilance accrue en ce qui concerne les activités des établissements financiers et de crédit iraniens afin d'empêcher le contournement du
} 
E. Castellarin, « Le gel des avoirs d'une banque centrale étrangère comme réaction à un illicite : rétorsion ou contre-mesure ? », Annuaire de La Haye de droit international, vol. 25, 2012, pp. 173-197

Le rapatriement d'avoirs, même à des personnes non ciblées par les mesures restrictives, entraîne en tout cas un risque de contournement : il est en effet impossible de connaître l'utilisation finale des avoirs rapatriés. Il résulte donc que la banque centrale peut utiliser ses avoirs seulement pour des activités souveraines (et pour les activités commerciales non interdites) dans l'Union européenne, mais non dans son Etat d'origine.

En d'autres termes, la nature sectorielle des dérogations suit une logique indépendante de celle de l'immunité d'exécution, qu'elles ne permettent pas de respecter dans tous les cas. La compatibilité avec l'immunité ne peut être assurée que par une dérogation générale concernant les avoirs affectés aux activités typiques de la banque centrale. Une catégorie de dérogations prévue par le Canada dans le cas de la Syrie semble inspirée par l'intention de concilier le gel des avoirs avec le droit international, puisqu'elle concerne les avoirs affectés à «toute activité exercée en application d'un accord ou d'un arrangement conclu entre le Canada et la Syrie $»{ }^{71}$ Cette disposition ne se réfère pourtant pas au droit coutumier et risque aussi de ne pas mettre l'exécutif dans la condition de toujours respecter l'immunité.

Toutes les formulations des dérogations démontrent donc que l'immunité coutumière n'a pas été prise en compte en tant que telle dans la conception des gels des avoirs. Puisque certains avoirs protégés par l'immunité font l'objet du gel, celui-ci est partiellement illicite en droit international et engage la responsabilité de l'Etat ou de l'Union européenne. La responsabilité est engagée indépendamment de la mise en œuvre effective du gel des avoirs, dès lors que la législation le prévoyant est seulement suspendue ou non appliquée mais produit encore des effets. $^{72}$

En outre, malgré les dérogations, les gels des avoirs des banques centrales d'Etats membres du Fonds monétaire international semblent prima facie incompatibles avec les droits que ces Etats tirent des Statuts du Fonds.

2. La compatibilité du gel des avoirs avec le droit du Fonds monétaire international

Le droit monétaire international protège la stabilité monétaire en interdisant certaines mesures restrictives des échanges internationaux. Par exemple, les Iranian Assets Control

\footnotetext{
présent règlement, notamment le gel des avoirs de la Banque centrale d'Iran. » (Règlement 267/2012, supra note 10, considérant 17).

${ }^{71}$ Règlement sur les mesures économiques spéciales visant la Syrie, supra note 13, art. 3.2, lett. a.

${ }^{72}$ Pour une hypothèque judiciaire suspendue mais non annulée : CIJ, supra note 27, par. 112.
} 
E. Castellarin, « Le gel des avoirs d'une banque centrale étrangère comme réaction à un illicite : rétorsion ou contre-mesure ? », Annuaire de La Haye de droit international, vol. 25, 2012, pp. 173-197

Regulations américaines de 1979 ont suscité plusieurs interrogations quant à leur nature présumée de « réglementation du contrôle des changes » au sens de l'art VIII section 2(b) des Statuts du FMI et à leur invocabilité devant les juridictions étrangères. ${ }^{73}$ Le gel des avoirs des banques centrales étrangères soulève à cet égard les mêmes questions que les gels d'avoirs individuels et les embargos. Toutefois, quoiqu'assorti de dérogations, il peut avoir des conséquences monétaires quantitativement plus importantes. ${ }^{74}$ Puisque son intérêt économique est de limiter l'activité commerciale de l'Etat visé dans des domaines stratégiques, il est conçu pour avoir un impact sur la balance des paiements et donc sur la stabilité de la monnaie de l'Etat visé. ${ }^{75}$ En effet, même quand le gel des avoirs permet à la banque centrale visée de poursuivre ses activités souveraines, la balance des paiements de son Etat en est affectée, car elle évolue selon les échanges commerciaux avec l'étranger indépendamment de la qualité publique ou privée des opérateurs économiques. L’Etat

${ }^{73}$ N. A. Simon, «The Iranian Assets Control Regulations and the International Monetary Fund Agreement: Are
the Regulations "Exchange Control Regulations"? », Boston College International and Comparative Law Review, vol. 4 (1981), pp. 203-223 ; W. F. Ebke, «Article VII, Section 2(b), International Monetary Cooperation, and the Courts », The International Lawyer, vol. 23 (1989), pp. 677-710.

${ }^{74}$ Malgré ses conséquences monétaires, l'embargo concernant l'or associé au gel des avoir des banques centrales (pour l'Iran, règlement 267/2012, supra note 10, art. 15 ; pour la Syrie, Décision 2012/739/PESC, supra note 12, art. 11) ne soulève pourtant pas de problèmes spécifiques quant au droit du FMI. Les Statuts ne comprennent plus, depuis le second amendement, aucune référence au commerce de ce métal (J. Gold, Legal and institutional aspects of the international monetary system : selected essays, Washington, FMI, vol. II, p 723-777). Il en va de même pour l'embargo sur les billets de banque (règlement 267/2012, supra note 10, art. 16 ; règlement 36/2012, supra note 12, art. 11) qui sont aussi des biens ordinaires dépourvus de valeur légale, certes nécessaires à l'activité de la banque centrale mais non jouissant d'un régime particulier en droit international.

${ }^{75}$ Il est difficile d'évaluer exactement l'impact monétaire du gel des avoirs par rapport aux autres mesures restrictives et à d'autres facteurs, mais les données économiques disponibles pour l'Iran fournissent un exemple de ce retentissement. Sans doute à cause de la progressive limitation de l'approvisionnement de devises étrangères, la devise iranienne, le rial, a subi une chute spectaculaire de son cours de marché. Le taux de change officiel fixé par le gouvernement iranien à environ 12000 rial pour un dollar est en effet réservé à certains opérateurs économiques; le gouvernement a introduit au profit des importateurs un taux fixe plus avantageux que le taux de marché pratiqué par les dealers de rue, qui peut atteindre 40000 rials pour un dollar (R. Gladstone, «A New Sign of Distress as Iran’s Currency falls », New York Times, $1^{\mathrm{er}}$ octobre 2012 ; Le Monde, «Le rial glisse et savonne la planche au gouvernement iranien », 2 octobre 2012). Ces pratiques sont surveillées par le FMI en tant que possibles taux de change multiples, interdits par l'art. VIII section 3 des Statuts, dont l'Iran a accepté les obligations en 2004. L'Iran a aussi accepté d'assurer la convertibilité officielle de sa monnaie selon l'art. VIII section 4. 
E. Castellarin, « Le gel des avoirs d'une banque centrale étrangère comme réaction à un illicite : rétorsion ou contre-mesure ? », Annuaire de La Haye de droit international, vol. 25, 2012, pp. 173-197

s'approvisionne donc plus difficilement en devises étrangères, qui lui sont nécessaires pour garantir la convertibilité de sa monnaie.

Empêchant l'accomplissement des opérations commerciales gérées par la banque centrale, le gel de ses avoirs est prima facie incompatible avec l'article VIII section 2(a) des Statuts du FMI (2.1). Toutefois, cette illicéité n'est que théorique, car la pratique de l'organisation permet de réputer licites les gels des avoirs adoptés par un Etat non pour équilibrer sa balance des paiements, mais pour des raisons de sécurité (2.2).

2.1 Le gel des avoirs comme restriction aux paiements relatifs à des transactions courantes

Le gel des avoirs affecte directement les opérations de commerce extérieur (2.1.1) en les restreignant : il est donc plus qu'un simple contrôle sur leurs modalités (2.1.2).

2.1.1. Le gel des avoirs comme mesure affectant des paiements pour transactions courantes

Selon l'article VIII section 2(a) des Statuts du FMI

«[s]ous réserve des dispositions de la section 3, paragraphe b), de l'article VII et de la section 2 de l'article XIV, aucun État membre n'impose, sans l'approbation du Fonds, de restrictions à la réalisation des paiements et transferts afférents à des transactions internationales courantes. »

Cet article, qui n'a pas été modifié lors des amendements aux Statuts et conserve une place centrale dans le système monétaire international ${ }^{76}$, protège la convertibilité dite "de marché" d'une devise, que l'on distingue de la convertibilité "officielle" opérant entre banques centrales. Tous les Etats ayant adopté jusqu'à présent des gels de avoirs de banques centrales étrangères ont accepté les obligations de l'article VIII. ${ }^{77}$ Le lien de cette disposition avec les objectifs du FMI est étroit, car elle permet d' «[a]ider à établir un système

\footnotetext{
${ }^{76}$ J. Gold, supra note 74, p. 171 et seq.; D. Carreau, Le Fonds Monétaire International, Paris, Pedone, 2009, p. 89 et seq. ; R. Geiger, "Legal Aspects of Convertibility », Georgia Journal of International and Comparative Law, vol. 4 (1974), pp. 74-92.

${ }^{77}$ Les Etats n'ayant pas accepté les obligations de l'art. VIII sont soumis au régime transitoire de l'art. XIV par. 2, selon lequel ils peuvent maintenir des restrictions aux paiements et transferts afférents à des transactions internationales courantes. La section 3, paragraphe b) de l'art. VII, concernant les monnaies rares, ne s'applique pas non plus aux gels d'avoirs actuellement en vigueur, qui ont pour objet des devises convertibles.
} 
E. Castellarin, « Le gel des avoirs d'une banque centrale étrangère comme réaction à un illicite : rétorsion ou contre-mesure ? », Annuaire de La Haye de droit international, vol. 25, 2012, pp. 173-197

multilatéral de règlement des transactions courantes entre les États membres »(iv), d'«abréger la durée et réduire l'ampleur des déséquilibres des balances des paiements des États membres»(vi) et par là de «[p]romouvoir la stabilité des changes» (iii) et de « [f]aciliter l'expansion et l'accroissement harmonieux du commerce international » (ii).

Les «paiements pour transactions courantes» sont définis par l'art XXX (d) des Statuts :

«Par paiements pour transactions courantes, il faut entendre les paiements qui n'ont pas pour objet le transfert de capitaux; ils comprennent notamment :

1) tous les paiements dus au titre du commerce extérieur et des autres opérations courantes, y compris les services, ainsi que les facilités normales à court terme de banque et de crédit;

2) les paiements dus au titre d'intérêts sur des prêts ou de revenus nets d'autres investissements;

3) les paiements d'un montant modéré pour l'amortissement d'emprunts ou la dépréciation d'investissements directs; et

4) les envois de fonds d'un montant modéré pour charges familiales. »

Cette définition, indépendante de la qualité officielle de celui qui accomplit ou reçoit le paiement, délimite la notion par rapport à celle de transfert de capitaux, soumis au régime différent et plus permissif de l'article VI section 3 des Statuts, qui en permet le contrôle, même de manière discriminatoire. ${ }^{78}$ En pratique, le contrôle des transferts de capitaux accompagne toujours le gel des avoirs des banques centrales dans le cadre d'un ensemble de mesures restrictives ciblant au moins une partie de l'économie d'un Etat.

\subsubsection{Le gel des avoirs comme restriction}

L'article VIII section 2(a) interdit les restrictions aux paiements pour transactions courantes, qu'ils soient faits vers l'étranger ou sur le territoire d'un Etat membre du FMI à un étranger souhaitant rapatrier les bénéfices ou les réutiliser dans un autre Etat membre du FMI.

Selon le Conseil des gouverneurs

${ }^{78}$ Décision n ${ }^{\circ}$ 541-(56/39), 25 juillet 1956 (FMI, Selected decisions, p. 510). 
E. Castellarin, « Le gel des avoirs d'une banque centrale étrangère comme réaction à un illicite : rétorsion ou contre-mesure ? », Annuaire de La Haye de droit international, vol. 25, 2012, pp. 173-197

"[t]he guiding principle in ascertaining whether a measure is a restriction on payments and transfers for current transactions under Article VIII, Section 2, is whether it involves a direct governmental limitation on the availability or use of exchange as such $\gg{ }^{79}$

L'inspiration historique de cette définition de restriction est liée aux mesures de portée générale, alors que le gel des avoirs des banques centrales, comme celui de personnes privées, n'opère qu'une restriction ad hoc; toutefois, la définition du Conseil des gouverneurs est suffisamment générale pour les inclure.

La restriction se distingue notamment du simple contrôle, qui consiste à établir les modalités du paiement sans l'interdire de manière générale. Ainsi, la soumission des paiements à des licences qui sont toujours octroyées ${ }^{80}$ ou à l'utilisation de certaines devises n'est pas considérée comme une restriction aux paiements. En revanche, l'interdiction faite à un opérateur économique de disposer de ses avoirs ne se borne pas à réguler les paiements, mais les empêche totalement. Ainsi, le gel des avoirs que les banques centrales utilisent pour des transactions courantes, mis en ouvre par l'exécutif et limitant la disponibilité de leurs avoirs, constitue une restriction. La définition du Conseil des gouverneurs se concentre sur la disponibilité des devises, mais est a fortiori remplie si tout type d'avoir est rendu indisponible. Les dérogations réduisent l'effet économique les gels d'avoirs, mais ne suffisent pas à rendre cette mesure un simple contrôle, car elles ne sont pas toujours octroyées par l'exécutif.

Prima facie, donc, le gel des avoirs des banques centrales exerçant des activités commerciales est contraire à l'article VIII section 2(a) des Statuts du FMI ; notamment, le fait qu'une restriction aux paiements pour transactions courantes a été adoptée pour des raisons de sécurité nationale ou internationale ne la justifie pas. Toutefois, la restriction est interdite seulement lorsqu'elle est accomplie «sans l'approbation du Fonds », qui est accordée de manière relativement large.

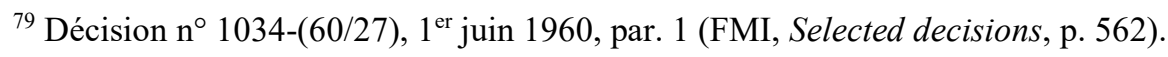

${ }^{80}$ J. Gold., supra note 74, p. 172.
} 
E. Castellarin, « Le gel des avoirs d'une banque centrale étrangère comme réaction à un illicite : rétorsion ou contre-mesure ? », Annuaire de La Haye de droit international, vol. 25, 2012, pp. 173-197

2.2. La licéité du gel des avoirs en raison de sa motivation

Le Fonds approuve tacitement les gels d'avoirs adoptés pour des raisons de sécurité (2.2.1), d'autant plus qu'ils ont des effets économiques limités sur le système monétaire international (2.2.2).

2.2.1 L'approbation tacite des restrictions aux paiements adoptés pour des raisons de sécurité

Généralement, les restrictions aux paiements sont utilisées, comme les contrôles de change et les restrictions aux transferts de capitaux, pour des raisons de balance commerciale. Du point de vue économique, l'effet d'une restriction aux paiements est indépendant de sa motivation, mais celle-ci se reflète sur la portée de la restriction. L'appréciation par le FMI de la licéité des restrictions adoptées uniquement pour des raisons de sécurité nationale ou internationale soulève les problèmes typiques des mesures restrictives ayant un contenu financier et une finalité politique : d'un côté, le Fonds est compétent en raison de la nature et des effets de la mesure ; de l'autre côté, il n'est pas le forum le plus adéquat pour en apprécier l'opportunité et les effets politiques. ${ }^{81}$ A l'égard des restrictions prises pour des raisons de sécurité la compétence du FMI doit donc s'exercer, mais d'une manière différente que pour les restrictions les plus communes, liées à la balance des paiements. Le Conseil des gouverneurs, conscient de cette difficulté, a poursuivi dès les premières années d'activité du Fonds une politique permissive fondée sur la non-interdiction plutôt que sur l'autorisation expresse.

Dans sa décision 144-(52/51), le Conseil des gouverneurs, tout en reconnaissant que la motivation d'une restriction n'a pas d'effet quant à sa licéité, reconnaît avant tout qu'il ne lui appartient pas d'établir la volonté de l'Etat adoptant la restriction; par conséquent, il reconnaît à chaque Etat membre la faculté de motiver certaines restrictions avec la préservation de la sécurité nationale ou internationale. Cette qualification unilatérale déclenche un régime juridique de faveur, caractérisé par une procédure d'approbation doublement spéciale, soumis à la condition de la notification des mesures au Fonds au plus tard 30 jours après leur adoption. A la différence des restrictions d'inspiration économique,

${ }^{81}$ R. W. Edwards, supra note 25, p. 874. 
E. Castellarin, « Le gel des avoirs d'une banque centrale étrangère comme réaction à un illicite : rétorsion ou contre-mesure ? », Annuaire de La Haye de droit international, vol. 25, 2012, pp. 173-197

qui doivent être expressément approuvées par le Conseil des gouverneurs, les restrictions justifiées par des raisons de sécurité profitent d'une présomption d'approbation: après examen par le Conseil des gouverneurs, si dans les 30 jours suivant la notification le Fonds n'informe pas l'Etat membre de la non-approbation des mesures, celles-ci sont réputées approuvées. ${ }^{82}$ La majorité requise pour l'adoption d'une décision doit donc se former contre les restrictions : tant que les mesures sont adoptées par des Etats détenant une large portion des quotes-parts, la majorité est politiquement impossible à atteindre. De surcroît, l'adoption d'une décision négative est de facto impossible à cause de l'utilisation de la règle du consensus.

Même en cas d'approbation tacite, les restrictions doivent être temporaires. ${ }^{83}$ Toutefois, leur maintien en vigueur procède d'un second élément de spécialité de la procédure d'évaluation : alors que le Fonds doit renouveler expressément l'approbation des restrictions d'inspiration économique, pour les restrictions adoptées pour des raisons de sécurité il se réserve simplement la faculté de révoquer ou modifier expressément l'approbation tacite après révision périodique. ${ }^{84}$

2.2.2 L'effet limité des gels des avoirs sur le système monétaire international

Jusqu'à présent, les gels des avoirs des banques centrales, régulièrement notifiés, ${ }^{85} \mathrm{ont}$ toujours rencontré l'approbation tacite du FMI. Si leur caractère temporaire n'est pas en question, ${ }^{86}$ la compatibilité de la procédure instituée par la décision 144-(52/51) avec les Statuts soulève des doutes. La validité des décisions du Conseil des gouverneurs est soumise au respect des Statuts, y compris de ses objectifs. ${ }^{87}$ Vue l'importance de l'interdiction des

\footnotetext{
${ }^{82}$ Décision n $^{\circ}$ 144-(52/51), 14 août 1952, par. 1 (FMI, Selected Decisions and Selected Documents of the IMF, Washingtion, Fonds monétaire international, $35^{\mathrm{ème}}$ éd., 2010, p. 556).

${ }^{83}$ Décision n ${ }^{\circ}$ 955-(59/45), 23 octobre 1959 (FMI, ibid., p. 560) ; J. Gold, « The Iran-United States Claims Tribunal and the Articles of the International Monetary Fund », George Washington Journal of International Law and Economics, Vol. 18, Issue 3 (1985), pp. 537-580, p. 575.

${ }^{84}$ Décision n ${ }^{\circ}$ 955-(59/45), ibid., par. 2.

85 FMI, Annual Report on Exchange Arrangements and Exchange Restrictions, $<$ http://www.elibrary.imf.org/page/AREAER/www.imfareaer.org>, consulté le 23 février 2013.

${ }^{86}$ Par exemple, Décision 2012/739/PESC, supra note 12, art. 31. Plus en général, les mesures restrictives sont temporaires car elles sont subordonnées au maintien par l'Etat ciblé de sa conduite.

${ }^{87}$ Statuts du FMI, art. préliminaire, i.
} 
E. Castellarin, « Le gel des avoirs d'une banque centrale étrangère comme réaction à un illicite : rétorsion ou contre-mesure ? », Annuaire de La Haye de droit international, vol. 25, 2012, pp. 173-197

restrictions aux paiements pour transactions courantes, une interprétation téléologique des Statuts aboutirait à considérer que, parmi les restrictions non prévues par les articles VII section 3(b) et XIV, seules celles temporaires et nécessaires pour rétablir la balance des paiements seraient admissibles par le Conseil des gouverneurs. ${ }^{88}$ Les mesures de sécurité, même tacitement approuvées par les Conseil des gouverneurs, seraient donc contraires à l'esprit des Statuts. ${ }^{89}$ Cette opinion semble bien fondée, car la procédure spéciale prévue par la décision 144-(52/51) peut être assimilée à un amendement des Statuts qui pourtant ne respecte pas les conditions prévues par l'article XXVIII des Statuts. ${ }^{90}$ Utilisée pour permettre l'application des résolutions du Conseil de sécurité, ${ }^{11}$ elle introduit aussi un risque d'arbitraire qui peut profiter aux seuls Etats ayant une participation majoritaire au Fonds.

Cette vision normativiste est toutefois à relativiser : l'analyse des restrictions par le FMI repose, selon la tradition de l'organisation, sur une logique moins strictement juridique. Les mesures restrictives sont appréciées dans le contexte de la politique économique des Etats qui les adoptent et de la situation économique mondiale. Il n'est pas tant question, en effet, de droits réciproques des Etats membres, mais de compatibilité des mesures avec le système monétaire international. L'esprit des Statuts, qui prima facie disqualifie toute restriction aux paiements, peut donc être utilement invoquée pour en tolérer certaines. Ainsi, par exemple, les mesures restrictives adoptées par les Etats-Unis sont appréciées dans le contexte général de leur participation satisfaisante au système international des paiements. ${ }^{92}$ De la même manière, les inquiétudes de l'Iran, ciblé par les restrictions aux paiements, ne sont partagées que dans la mesure où elles reflètent la dégradation possible du commerce international de pétrole. ${ }^{93}$ De

\footnotetext{
${ }^{88}$ v. M. R. Schuster, M. Robert, The Public International Law of Money, Oxofrd, Clarendon press, 1973, p. 155.

${ }^{89}$ N. A. Simon, supra note 73, p. 221.

${ }^{90}$ Outre l'approbation du Conseil des gouverneurs, cet article requiert les trois cinquièmes des États membres disposant de quatre-vingt-cinq pour cent du nombre total des voix.

${ }^{91}$ Le premier précédent de ce type est celui des transactions concernant la Rhodésie en 1966 (J. Gold, Membership And Nonmembership In The International Monetary Fund, Washington, Fonds monétaire international, 1974, pp. 277-78).

${ }^{92}$ FMI, Staff report dans le cadre des consultations avec les Etats-Unis prévues par l'art. IV des Statuts, 2 août 2012, p 65. Les mesures de l'Union européenne et du Canada n'ont pas encore été prises en considération dans le même cadre.

${ }^{93}$ FMI, Public Information Notice dans le cadre des consultations avec l'Iran prévues par l'art. IV des Statuts, 2011 : «Many Directors noted the authorities' concern about the impact of sanctions on the transfer of oil export proceeds, including potential adverse effects on oil markets, and some Directors called on the staff to monitor and assess Iran's access to the international payment system. » Toutefois, cette analyse est antérieure au
} 
E. Castellarin, « Le gel des avoirs d'une banque centrale étrangère comme réaction à un illicite : rétorsion ou contre-mesure ? », Annuaire de La Haye de droit international, vol. 25, 2012, pp. 173-197

manière plus générale, la pratique des restrictions aux paiements semble soulever peu d'intérêt par rapport à d'autres sujets en raison de son faible impact sur l'économie mondiale, puisque la place centrale de l'article VIII section 2(a) dans les Statuts était inspirée par la nocivité des restrictions généralisées dans l'entre-deux-guerres.

Du point de vue de la viabilité du système monétaire international, l'effet le plus grave des restrictions aux paiements courants se produit lorsqu'elles sont appliquées par un Etat membre de manière généralisée et sans dérogation. Il ne s'agit pas, en effet, d'une pratique dont la nocivité économique est due à son caractère discriminatoire, à la différence des pratiques visées par l'article VIII section 3, y compris les taux de change multiples. En cela, elles semblent plutôt assimilables à la restriction des mouvements de capitaux (article VI section 3), dont la licéité n'est pas exclue par leur caractère discriminatoire. En d'autres termes, le caractère discriminatoire de ces restrictions contribue paradoxalement à son acceptation par le FMI : leur impact jusqu'à présent minime sur le système des paiements internationaux a permis d'éviter largement la controverse sur leur licéité. Par conséquent, en dépit de sa nature restrictive des paiements pour transactions courantes, le gel des avoirs des banques centrales étrangères n'est pas considéré comme illicite en droit du FMI.

\section{Conclusion}

Par l'adoption de gels des avoirs de banques centrales étrangères, l’Union européenne et le Canada ont franchi une étape dans le durcissement de leurs mesures restrictives, imitant ainsi les Etats-Unis. Ces mesures, toujours adoptées à cause des fonctions accomplies par les banques centrales, s'inscrivent dans une stratégie active de réaction décentralisée aux faits internationalement illicites, même en l'absence d'une autorisation du Conseil de sécurité quant à leur adoption. Elles s'appuient sur des normes permissives et utilisent un levier économique pour poursuivre des buts politiques; toutefois, elles peuvent être illicites in se. Leur compatibilité avec le droit du FMI est assurée par l'autorisation tacite du Conseil des gouverneurs ; en revanche, la manière dont sont formulées la plupart des dérogations aux gels des avoirs en vigueur ne suffit pas à exclure la saisie d'avoirs affectes à des activités souveraines et protégés par une immunité coutumière.

durcissement des mesures restrictives de 2012. Pour la Syrie, aucune analyse similaire n'est disponible car les dernières consultations dans le cadre de l'art. IV des Statuts se sont tenues en 2009. 
E. Castellarin, « Le gel des avoirs d'une banque centrale étrangère comme réaction à un illicite : rétorsion ou contre-mesure ? », Annuaire de La Haye de droit international, vol. 25, 2012, pp. 173-197

Réagissant à un fait internationalement illicite, les gels des avoirs des banques centrales étrangères actuellement en vigueur doivent donc être qualifiés de contre-mesures et non de simples rétorsions. Par conséquent, ils peuvent être adoptés par un sujet lésé par l'illicite auquel ils réagissent. En revanche, est discutée la question de savoir si un sujet non lésé est aussi habilité à les adopter. Leur licéité peut être exclue seulement aux conditions restrictives codifiées aux articles 49-53 du projet de la CDI sur la responsabilité des Etats. L'évaluation du respect de ces conditions ne peut qu'être faite au cas par cas : elle soulève notamment la question de la proportionnalité par rapport à l'illicéité d'une mesure qui marque une évolution des "sanctions intelligentes" des années 2000. 